\title{
Diabetic retinopathy and pancreas transplantation: a 3-year follow-up
}

\author{
A. Scheider ${ }^{1}$, E. Meyer-Schwickerath ${ }^{1}$, J. Nusser ${ }^{2}$, W. Land ${ }^{3}$ and R. Landgraf ${ }^{2}$ \\ Departments of ${ }^{1}$ Ophthalmology, ${ }^{2}$ Internal Medicine Innenstadt and ${ }^{3}$ Transplantation Center Klinikum Grosshadern, \\ Ludwig-Maximilians-University, Munich, FRG
}

Summary. The effect of simultaneous pancreas and kidney transplantation on diabetic retinopathy was studied in a prospective study with 30 patients (57 eyes) and 15 control subjects ( 26 eyes), patients who lost the pancreas, but preserved kidney function. There was no significant difference between the groups after a mean observation time of more than 35 months (a range of 12 to 96 months). Both populations had a stable retinopathy during follow-up. This seems to be a consequence of the far advanced retinopathy (mean duration of type 1 diabetes was 22 years) and the high percentage of coagulated eyes ( $81 \%$ and $85 \%$, respectively), but is not related to the organ transplantation. A closer look at the few patients who did not receive laser coagulation (14 patient and 6 control eyes), produced a different result. Four control eyes experienced a significant deterioration of the retinopathy which had been stable before rejection. It is the most important and so far never mentioned aspect of this study, that periods of destabilisation are a definite threat for the retinopathy. Nevertheless, it seems questionable whether we will ever be able to make a definite statement on the pancreas-eye relation, as long as the transplantation must be restricted to carefully selected late-stage diabetic subjects.

Key words: Diabetes mellitus - pancreas transplantation - diabetic retinopathy

\section{Introduction}

In 1988, Klein and colleagues published the results of a four year study on 891 younger- and 987 older-onset diabetic persons concerning the connection between hyperglycemia and retinopathy and concluded that their data suggest a strong and consistent relationship for incidence and progression of retinopathy after controlling for duration of diabetes, age, sex, and baseline retinopathy. (Klein et al. 1988). So far, published results suggest that a successful pancreas transplantation is superior to any other form of conventional diabetes treatment in achieving normoglycaemia, and it should therefore be the best means of preventing or halting diabetic retinopathy (Bolinder and Tyden 1989). This assumption is supported by the fact, that simultaneously transplanted kidneys do not show significant diabetic changes as long as pancreatic grafts work well (Bilous et al. 1989).

The data of participating ophthalmologists were controversial in the first period. Ramsay studied the effect of successful pancreas transplantation and consequent normoglycaemia on visual function and diabetic retinopathy in 22 patients with Type 1 (insulin-dependent) diabetes mellitus. Sixteen similar patients in whom pancreas transplantation had been unsuccessful served as a control group. The majority of patients in both groups had advanced proliferative retinopathy. At a mean followup of 24 months Ramsay found no significant difference between the groups in the rate of progression of retinopathy and concluded that success of the transplantation did not prevent progression of retinopathy across the range of retinopathy studied (Ramsay et al. 1988).

Nevertheless, Kampik and Ulbig from our institution reported in an earlier study, that $46 \%$ of a group of transplanted patients had unchanged and $32 \%$ improved visual acuity (Ulbig et al. 1987). They concluded that the simultaneous transplantation has a favourable effect on diabetic retinopathy. The main concern in this study was the lack of a control group. Though the visual acuity is the most important factor for the patient, it depends on many others which are not all related to the retinopathy, such as patient compliance, refraction, lens opacities or optic nerve dysfunctions. Some ophthalmologists therefore question the usefulness of the eye as a satisfactory organ to judge the effect of pancreas transplantation (Klein 1988).

It is our impression, that most patients who come to the first pre-transplant evaluation, wish to improve their visual acuity by means of this operation. This was the main reason for us to re-evaluate our data two years ago and to compare them with a control group of patients who lost their pancreas but preserved kidney function. We came to the conclusion that there was no difference in outcome 
between 24 patients and 7 control subjects, but in contrast to Ramsay we did not see a progression of retinopathy (Scheider et al. 1989). Such results raise the question, wether we use the appropriate control group, a major concern of the centres which favour the single kidney transplantation.

Recently, Petersen and colleagues published a small study on four patients after simultaneous pancreas and kidney transplantation compared with four control subjects (Petersen et al. 1990). They found a steady progression in both groups. This article is discussed by R.N. Frank, Detroit, who pointed out the major problems of all hitherto mentioned publications. He mentions that the number of subjects is too small, the observation period too short and that most patients have far advanced retinopathy, the same concerns which we published in our two year report (Scheider et al. 1989).

This time, after nearly four years, we can evaluate the hitherto largest population with the longest observation time ever in patients who have undergone transplantation. Due to the above-mentioned problems, we decided to concentrate on patients who did not receive laser coagulation and who lost their transplant after a stable period. We presumed that a change in retinopathy after rejection could be further proof of a possible benefit of pancreas transplantation.

\section{Subjects and methods}

The presented results are calculated from all patients with a regular follow-up of at least 12 months and where a reasonable fundus picture quality could be achieved. Out of the group of patients who had received a simultaneous pancreas and kidney transplantation in Munich since may 1982, 42 fulfilled these criteria. 12 of the 42 , who had lost their pancreatic graft, but had a well functioning kidney, served as a control group. Three patients changed group during follow-up because of a late rejection of the pancreas.

All patients had a regular ophthalmological examination every 6 to 12 months. This included best corrected visual acuity, applanation tonometry, slitlamp examination and dilated binocular funduscopy with $20^{\circ}$ and $90^{\circ}$ lenses. Seven 30 degree fundus pictures of the posterior pole were taken. The images were graded by comparing them with the ETDRS (Early Treatment Diabetic Retinopathy Study Group)-standard photographs. Since we did not take stereoscopic photographs, the original Airlie House grading scale had to be changed, as noted in Table 2. All photographs were independently evaluated by two of us (A.S. and E.M-S.). Since photographs in late stage diabetic patients are often difficult to evaluate even where an ophthalmological examination is possible (small pupil due to diabetic neuropathy, beginning cataract), we developed a three-page grading protocol, in which all anterior and posterior segment structures could subjectively be graded during examination. This was done independently by two examiners (A.S. and E.M-S.). Fluorescence angiographies were taken in all patients with unexplained visual loss or questionable neovascularisations. In visual acuity, a decrease of two or more lines was considered to be significant. All results were entered into a computer for further evaluation.

DR Score: $R \quad 0$ = no retinopathy, $R$ I = mild nonproliferative disease (Microaneurysms, dot haemornages), R 2 = preproliferative disease (nerve fiber layer infarcts, venous beading, intraretinal microangiopathy), R 3 = proliferative retinopathy (pr) with one high risk factor (=hrf: neovascularisation on the disc (nvd) or elsewhere (nve), large nv, preretinal haemorrhage), R $4=$ pr with two hrf or nvd, $R 5=3 \mathrm{hrf}, \mathrm{R} 6=4 \mathrm{hrf}, \mathrm{R} 7=$ mild vitreous traction with active nv's, $R \mathbf{8}=$ strong traction with active nv's, $R 9=$ mild fibrous traction, $R \quad 10=$ strong fibrous traction, $R 11=$ no evaluation possible.
For statistical analysis, we used the paired two-tail $t$-test and the nonparametric Wilcoxon signed rank test for smaller subject samples.

\section{Results}

The study group consists of 30 patients with well functioning grafts, normoglycaemia without further insulin therapy and a mean $\mathrm{HbA}_{1}$ of $7.15 \pm 0.92 \%$. The mean creatinin level was $1.46 \pm 0.33 \mathrm{mg} / 100 \mathrm{ml}$, mean blood pressure 146 to 91 (Table 1).The mean age was 37 \pm 7 years with a range from 27 to 53 years and a 22 -year history of diabetes mellitus prior to transplantation. The observation time was $38 \pm 21$ month (a range of 12 to 96 months). Since three patients had one irreversibly blind eye, 57 eyes could be evaluated. The average preoperative visual acuity was measured to be $0.51 \pm 0.29$ (range from fingercounting to 1.0 ) with a retinopathy score of $4.33 \pm$ 3.79 (ranging from 0 to 11 ), which corresponds to a proliferative state. $81 \%$ of the patients had been treated with panretinal laser coagulation prior to transplantation. After the mean observation time of 38 months, we measured a best corrected visual acuity of $0.5 \pm 0.3$ and a retinopathy score of $3.95 \pm 3.5$ which means no significant change (Table 2, Fig. 1).

The visual acuity decreased in 5 eyes (9\%). Reasons were increasing lens opacification in three patients; macular oedema and anterior ischemic optic neuropathy in one patient. 5 eyes $(9 \%)$ had an increase in visual acuity due to cataract extraction in two and clearing of a vitreous haemorrhage in one patient. In the other two, a reason could not be documented. Improved retinopathy scores were due to clearing of vitreous haemorrhages in three and due to regression of proliferations in one patient. One patient suffered from a second vitreous hemorrhage.

Table 1. General patient data.
Study group

Nr. of Patients:

Mean age:

Diabetes duration (yrs): $22 \pm 6$

Mean $\mathrm{HbA}_{1}$ (\%):

$7.15 \pm 0.92$

Mean creatinin (mg):

$1.46 \pm 0.33$

Mean blood pressure:

146 to 91
Control group

15

$39 \pm 7$ years

$22 \pm 5,1$

$8.39 \pm 1.78$

$1.3 \pm 0.23$

141 to 91
The 15 control patients had a mean age of $39 \pm 7$ years (31 to 55 years) and a 22-year history of diabetes mellitus. They all were insulin dependent. Their mean $\mathrm{HbA}_{1}$ was $8.39 \pm 1.78 \%$, the mean creatinin level $1.3 \pm$ $0.23 \mathrm{mg} / 100 \mathrm{ml}$ and the mean blood pressure 141 to 91 (Table 1). Since four eyes were irreversibly blind, 26 eyes could be studied of which $85 \%$ had received panretinal 0.34 (finger-counting to 1.0 ) and the retinopathy score was $4.42 \pm 3.29$ ( 1 to 10 ). After a mean follow-up of 35 (13 to 86) months after pancreas rejection, we measured a 
Table 2. Ophthalmological patient data.

\begin{tabular}{|c|c|c|c|c|c|}
\hline & \multicolumn{5}{|c|}{ Sudy Group } \\
\hline & $\begin{array}{l}\text { Observation } \\
\text { time (months) }\end{array}$ & $\mathrm{VA}^{1}$ & $\begin{array}{l}\mathrm{VA}^{1} \\
\text { Post } \mathrm{Op}_{\mathrm{p}}\end{array}$ & $\begin{array}{l}\mathrm{DR}^{2} \\
\text { Score } \\
\end{array}$ & $\begin{array}{l}\mathrm{DR}^{2} \text { Score } \\
\text { post Op }\end{array}$ \\
\hline Mean: & 37.91 & 0.51 & 0.51 & 4.33 & 3.95 \\
\hline \pm & 21.53 & 0.29 & 0.29 & 3.79 & 3.51 \\
\hline Min: & 12 & 0 & 0 & 0 & 0 \\
\hline \multirow[t]{3}{*}{ Max: } & 96 & 1 & 1 & 11 & 11 \\
\hline & \multicolumn{5}{|c|}{ Control Group } \\
\hline & $\begin{array}{l}\text { Observation } \\
\text { time (months) }\end{array}$ & $\mathrm{VA}^{1}$ & $\begin{array}{l}\text { VA }^{1} \\
\text { Post Op } \\
\end{array}$ & $\begin{array}{l}\mathrm{DR}^{2} \\
\text { Score } \\
\end{array}$ & $\begin{array}{l}\mathrm{DR}^{2} \text { Score } \\
\text { post Op }\end{array}$ \\
\hline Mean: & 34.35 & 0.54 & 0.50 & 4.12 & 4.54 \\
\hline \pm & 23.6 & 0.31 & 0.35 & 3.23 & 3.24 \\
\hline Min: & 13 & 0 & 0.02 & 0 & 0 \\
\hline Max: & 86 & 1 & 1 & 11 & 10 \\
\hline
\end{tabular}

visual acuity of $0.58 \pm 0.33$ (fingercounting to 0.9 ) and a retinopathy score of $4.25 \pm 3.22$ (1 to 10 ) (Table 2, Fig. 2). Again, these differences were not significant.

Five eyes had a significant change in visual acuity. Vision decreased in one eye due to lens opacification, while it increased in two because of cataract extraction. No reason could be documented in the other. The case with improved retinopathy score was due to cataract extraction. The eyes which deteriorated belonged to two patients who refused laser coagulation.

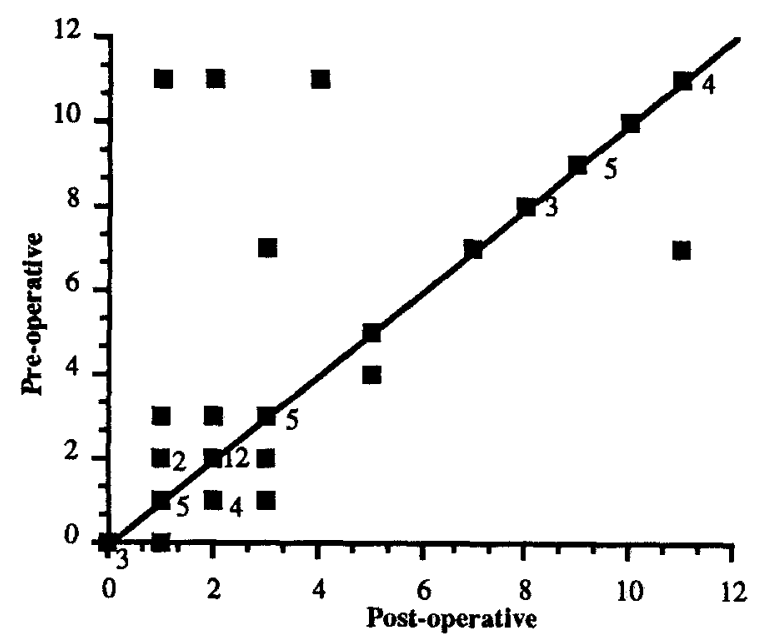

Fig. 1. Diabetic retinopathy score of all patient eyes. Signs above the diagonal indicate an improvement. When signs indicate more than one eye, the number is mentioned.

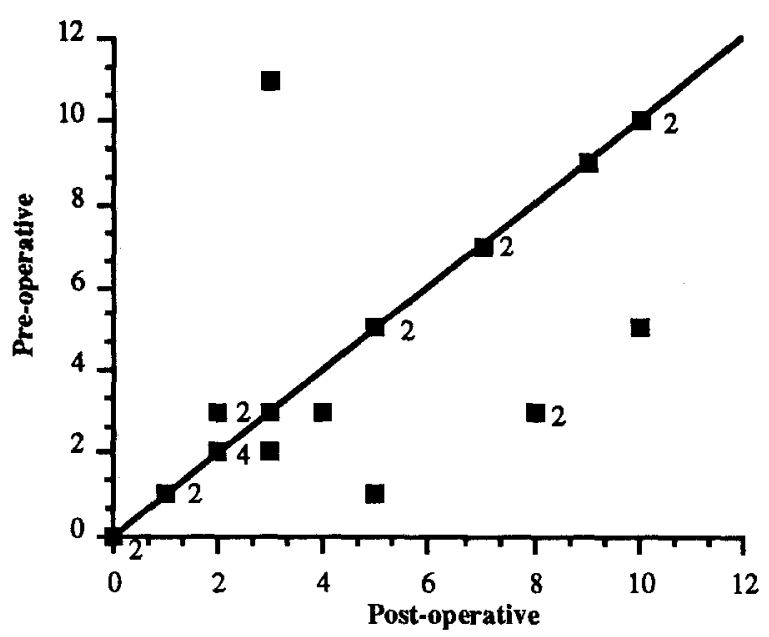

Fig. 2. Diabetic retinopathy score of all control eyes. Signs above the diagonal indicate an improvement. When signs indicate more than one eye, the number is mentioned.

\section{4-year follow-up}

A considerable number of subjects could be followed longer than 30 months ( 27 in the study group and 13 in the control group with a mean follow-up of 52 months). Since these are still more eyes then presented in most other studies, we calculated the results of this subgroup as a 4-year follow-up. Table 3 shows that there is however no significant difference between the groups even for longer observation times.

Table 3. Patients with observation times longer than 30 months

\begin{tabular}{|c|c|c|c|c|c|}
\hline & \multicolumn{5}{|c|}{ Study Group } \\
\hline & $\begin{array}{l}\text { Observation } \\
\text { time (months) }\end{array}$ & $\mathrm{VA}^{1}$ & $\begin{array}{l}V^{1} \\
\text { Post Op }\end{array}$ & $\begin{array}{l}\mathrm{DR}^{2} \\
\text { Score } \\
\end{array}$ & $\begin{array}{l}D^{2} \text { Soore } \\
\text { post Op }\end{array}$ \\
\hline Mean: & 52.07 & 0.57 & 0.58 & 3.93 & 3.50 \\
\hline \pm & 20.32 & 0.28 & 0.31 & 3.61 & 3.26 \\
\hline Min: & 30 & 0 & 0.01 & 0 & 0 \\
\hline \multirow[t]{3}{*}{ Max: } & 96 & 1 & 1 & 11 & 9 \\
\hline & \multicolumn{5}{|c|}{ Control Group } \\
\hline & $\begin{array}{l}\text { Observation } \\
\text { time (months) }\end{array}$ & $\mathrm{VA}^{1}$ & $\begin{array}{l}\mathrm{VA}^{1} \\
\text { Post } \mathrm{Op}_{\mathrm{p}}\end{array}$ & $\begin{array}{l}\mathrm{DR}^{2} \\
\text { Score } \\
\end{array}$ & $\begin{array}{l}\mathrm{DR}^{2} \text { Score } \\
\text { post Op }\end{array}$ \\
\hline Mean: & 46.79 & 0.54 & 0.52 & 5.57 & 5.07 \\
\hline \pm & 26.18 & 0.35 & 0.33 & 3.39 & 3.02 \\
\hline Min: & 13 & 0 & 0.03 & 2 & 2 \\
\hline Max: & 86 & 1 & 1 & 11 & 10 \\
\hline
\end{tabular}


Outcome of eyes without laser coagulation after pancreas rejection

We also examined a small group of patients who had not received laser coagulation; altogether 14 patient and 6 control subject eyes. The 6 control subject eyes belong to three patients, where a period of good transplant function (12, 17 and 33 months) could be compared with a longer period after pancreas rejection. These three are therefore included in both groups and make an intraindividual follow-up possible. As depicted in figure 3 and 4 , the mean retinopathy score remains stable in the patient group, but increases 2.8 points in the control group. The deterioration was caused by two patients who experienced a sudden onset of massive proliferation during the first two years after rejection (Fig. 3, 4). Due to the small subject number, an effective statistical evaluation was not possible.

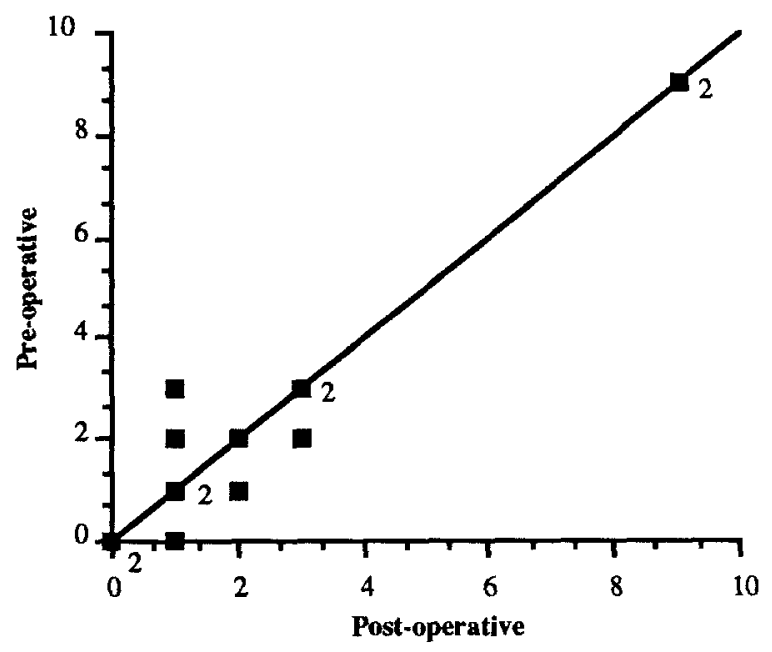

Fig 3. DR score of all patient eyes without laser coagulation. Signs above the diagonal signify improvement. When signs indicate more than one eye, the number is mentioned.

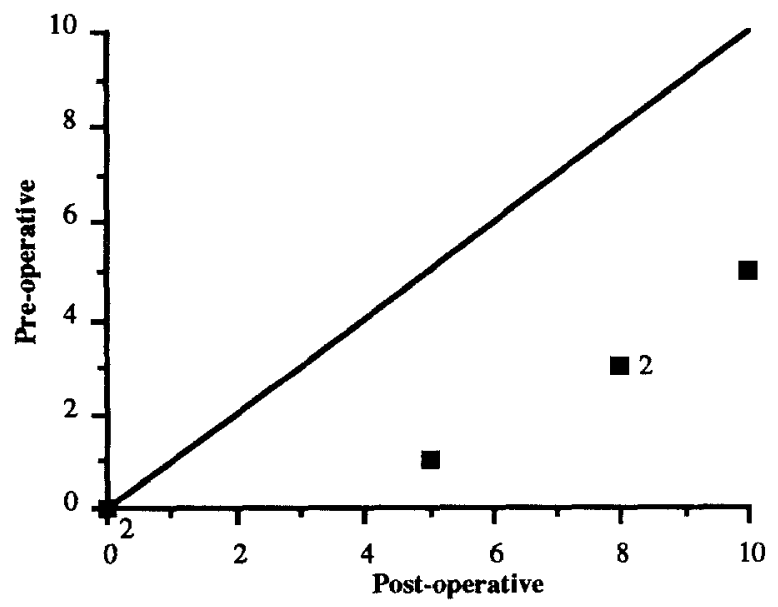

Fig 4. DR score of all control eyes without laser coagulation. Signs above the diagonal signify improvement. When signs indicate more than one eye, the number is mentioned.

\section{Discussion}

In contrast to some early positive reports (Ulbig et al. 1987), later publications conclude that pancreas transplantation does not influence the course of diabetic retinopathy when compared with a control group (Ramsay et al. 1988, Scheider et al. 1989, Petersen et al. 1990). In our first report, we concluded that larger patient populations and longer observation times are probably necessary, to find a statistically significant difference. This was expected from the paper of Ramsay in 1988, who reported this as a future possibility for his study group.

This study, which is now the largest so far published, concludes with an $90 \%$ stabilisation or improvement, which is also true for the controls. Even if we only compare the eyes with an observation time of more then 30 months, no significant differences can be measured (Table 3). Most significant changes of retinopathy score or visual acuity are due to clearing or clouding of the optical pathway and not related to the retina.

Several points should be mentioned, which are responsible for the lack of difference between the groups.

1) The most important fact is that $80 \%$ of our patients had received a panretinal laser coagulation prior to transplantation. This means that the observed stable condition in both groups over time might only underline the efficiency of this treatment, which has been proven in many studies (DRS 1987, ETDRS 1987, Koerner 1987). The destruction of 30 to $80 \%$ of all retinal tissue leads to a low but stable function of the retina. It is obvious that scar tissue cannot be ameliorated. A true reversal of retinal changes is unlikely once the preproliferative state has been passed.

2) The mean duration of diabetes before transplantation was 22 years in both groups. It is well known, that most patients experience their earliest changes within the fifth year of diabetes (Burger et al. 1986). The median annual rate of progression was found to be $12-13 \%$, independent of the previous duration of diabetes and the actual retinal state. $50 \%$ of all patients will have retinal changes ten years after onset of juvenile diabetes mellitus.

3) The mean $\mathrm{HbA}_{1}$ value was only slightly worse in the control group and better then 10 , which seems to be an upper limit for the prevention of progression of diabetic retinopathy, as Klein could demonstrate in 1988 (Klein et al. 1988).

4) This raises the question of whether we have the right control group. All our patients are highly motivated and very well monitored and are therefore not representative of the average, probably less concerned diabetic patient who might have more problems to stabilize his/her $\mathrm{HbA}_{1}$ level. On the other hand, post-transplantation check-ups are time-consuming for most patients. We know that we do not see all patients. Do we see only the patients with good compliance who might be those with better results? It has to be mentioned that we observed twice an unexplained significant visual loss immediately after transplantation, most probably due to anterior ischemic optic neuropathy. These patients are not included in our evaluation, since they never reached a one-year follow-up. 
Arguments 1,2 and 3 lead us to the conclusion that pancreas transplantation cannot be expected to have an effect in patients with a proliferative retinopathy, especially after laser coagulation. If patients could be transplanted between the fifth and tenth year after the onset of diabetes, we would have a realistic chance of judging the effect of pancreas transplantation.

Since this does not seem to be possible in the near future, we examined more closely the small group of patients who had never received laser coagulation. Included are three patients, where a period of good transplant function could be compared with a longer period after rejection. This made a intraindividual follow-up possible. Ocular changes, not related to the pancreas transplantation should therefore be minimized. The surprise was that the only two patients of the entire study with a marked decrease of the retinopathy score both belong to this group of three patients with a period of good transplant function before rejection. These figures at least indicate that something might happen during the first two years after rejection, which could be responsible for the progression. The main question remains; whether we have to search for positive effects on the study group or for negative effects on the control group? Are there so far unknown positive relationships between the pancreas and the eye? Are patients who experience multiple rejection episodes treated with more aggressive medications than the average patient and do these rejection episodes aggravate the retinopathy? Or are these deteriorations a consequence of the renewed insulin treatment? The latter seems most likely. It is well known that tighter control of the blood sugar can deteriorate the retinopathy (Rosenlund et al. 1988).

It is the most important and so far never mentioned aspect of our study, that these periods of destabilisation are a definite threat to the retinopathy. The study proves further that a longer observation time does not change the results of hitherto published studies. The pancreas transplantation has no positive effect on late-stage diabetic retinopathy.

In conclusion: We presented the largest study with the longest observation time, but could not solve the two major problems: 1 . Small study groups. The results of one or two patients have strong influences on the results of the whole group. 2 . The proliferative eye. It is certainly not the right organ to study the effect of pancreas transplantation. Possible advantages could probably only be visible for the ophthalmologist if we were able to compare large numbers of eyes with minimal and therefore still reversible retinal changes. The ETDRS needed more then 3000 patients in each group to find a significant advantage for laser coagulation (ETDRS 1987). It seems therefore questionable whether we will ever be able to make a definite statement on the pancreas-eye relation, as long as the transplantation must be restricted to a few carefully selected late-stage diabetic subjects. But we must conclude from this study, that no positive relationship can so far be seen.

\section{References}

Bilous RW, Mauer SM, Sutherland DER, Steffes MW (1989) The effect of pancreas transplantation on the glomerular structure of renal allografts in patients with insulin-dependent diabetes. New England Journal of Medicine 321:80-85

Bolinder J, Tyden G (1989) Indication, selection of patients and timing for pancreatic transplantation. Baillière's Clinical Gastronetreology 3:825-833

Burger W, Weber B, Hartmann R, Hovener G (1986) Annual progression of retinopathy in conventionally treated children and adolescents with type I diabetes mellitus. Acta Endocrinol Suppl (Copenh)

DRS (1987) Indications for photocoagulation treatment of diabetic retinopathy: Diabetic Retinopathy Study Report no. 14. The Diabetic Retinopathy Study Research Group. Int Ophthalmol Clin 27:239-53

ETDRS (1987) Treatment techniques and clinical guidelines for photocoagulation of diabetic macular edema. Early Treatment Diabetic Retinopathy Study Report Number 2. Early Treatment Diabetic Retinopathy Study Research Group. Ophthalmology 94:761-74

Klein R (1988) Discussion of: Landgraf, R. Landgraf-Leurs, M.M.C. Kampik, A. et al.: Pancreatic transplantaion as a means for prevention or treatment of late diabetic complications? Pediat. adolesc. Endocr. 17:235-243

Klein R, Klein BE, Moss SE, Davis MD, DeMets DL (1988) Glycosylated hemoglobin predicts the incidence and progression of diabetic retinopathy. Jama 260:2864-71

Koerner F (1987) [Possibilities and limitations of photocoagulation treatment in diabetic retinopathy]. Ther Umsch 44:273-82

Petersen MR, Vine AK, Committee TUoMPTE (1990) Progression of diabetic retinopathy after pancreas transplantation. Opthalmology 97:496-502

Ramsay RC, Goetz FC, Sutherland DE, Mauer SM, Robison LL, Cantrill HL, Knobloch WH, Najarian JS (1988) Progression of diabetic retinopathy after pancreas transplantation for insulindependent diabetes mellitus. $N$ Engl J Med 318:208-14

Rosenlund EF, Haakens K, Brinchmann HO, Dahl JK, Hanssen KF (1988) Transient proliferative diabetic retinopathy during intensified insulin treatment. Am J Ophthalmol 105:618-25

Scheider A, Thurau S, Ulbig M, Meyer-Schwickerath E (1989) Diabetic retinopathy remains stable after combined kidney and pancreas transplantation for type I diabtes mellitus. Diabetologica 32:538A

Ulbig M, Kampik A, Landgraf R, Land W (1987) The influence of combined pancreatic and renal transplantation on advanced diabetic retinopathy. Transplant Proc 19:3554-3556

Dr. A. Scheider

Department of Ophthalmology

University of Munich

Mathildenstr. 8

8000 Munich 2

FRG 